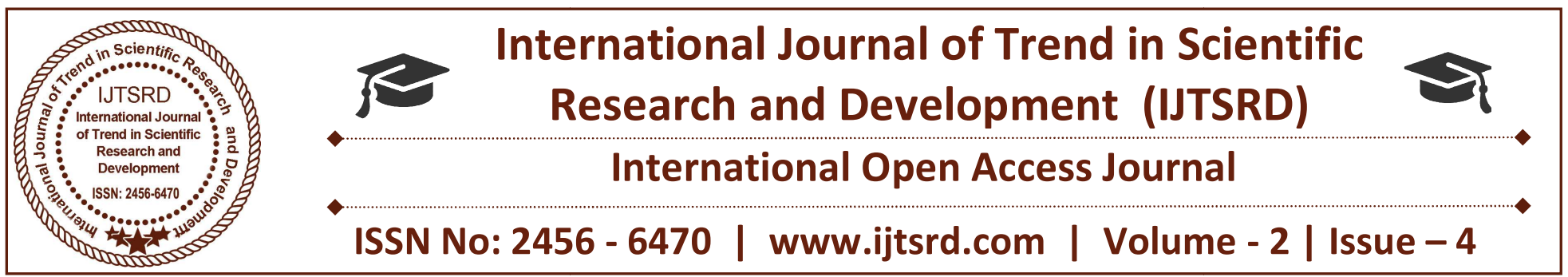

\title{
A Review on the Antimicrobial Activity of Sesuvium Portulacastrum
}

\author{
G. Beulah ${ }^{1}$, D. Divya ${ }^{2}$, Dr. G. Simhachelam ${ }^{3}$ \\ ${ }^{1}$ Lecturer in Zoology, AP Model Jr. College, Guntur, Andhra Pradesh, India \\ ${ }^{2}$ Lecturer in Zoology, St. Anns College for Women, Hyderabad, Telangana, India \\ ${ }^{3}$ Associate Professor, Acharya Nagarjuna University, Guntur, Andhra Pradesh, India
}

\begin{abstract}
Mangroves are unique group of vascular plants that occur in saline coastal habitats and are known to tolerate extreme environmental conditions. Some mangrove plants are used for a wide range of conditions, including bacterial, fungal, and viral diseases. The rise of antibiotic-resistant microorganisms is one of the severe problems in healthcare systems of the world, and infectious diseases are the second most serious cause of death worldwide. Therefore, new drugs have to be found in order to combat such diseases and it is essential to find new compounds that have antimicrobial properties. Medicinal-plant extracts, known to produce certain bioactive molecules which react with other organisms in the environment, are known to be less toxic to humans and are environmentally friendly due to the less pollutant released during production. Antimicrobial properties of medicinal plants are being increasingly reported worldwide. Mangroves are biochemically unique and produce a wide array of novel natural products and are considered a rich source of steroids, triterpenes, saponins, flavonoids, alkaloids, and tannins. Extracts from the leaves, stems, barks, and roots of mangrove species have shown positive results for antioxidant activity tests.
\end{abstract}

Keywords: Sesuvium, Bio active compounds, Antibacterial activity

\section{INTRODUCTION}

Sesuvium portulacastrum also known as shoreline purslane or sea purslane is a sprawling perennial herb that can exist under stress conditions growing up to $30 \mathrm{~cm}$ tall.

Preliminary phytochemical screening of leaf showed the presence of alkaloid, coumarin, flavonoid, phenol, steroid, tannins, terpenoid, and sugar in the different extracts. The plant possess broad spectrum activity against gram positive and gram negative bacteria as well as significant antifungal and antioxidant activity. It possess a number of bioactive constituents like Phytol, Squalene, Vitamin E, Benzoic acid, Hexadecanoic acid, ethyl ester, Oleic acid, eicosyl ester.

\section{DESCRIPTION OF THE PLANT:}

There are twelve different species in the genus Sesuvium which are distributed in different parts of globe. Sesuvium portulacastrum, was first published in 1953 as Portulaca portulacastrum by Carl Linnaeus.. As it is well conform to salinity and drought, Sesuvium portulacastrum is known to be halophytic species. It is a frequent pioneer species in the backshore zone of coastal beaches, where sand movement is influenced by prevalent winds near the born crest.

\section{TAXONOMIC ARRANGEMENT}

Kingdom : Plantae

Subkingdom : Viridiplantae

Division : Tracheophyta

Subdivision : Spermatophytina

Class : Magnoliopsida

Superorder : Caryophyllanae

Order : Caryophyllales

Family : Aizoaceae

Genus : Sesuvium

Species : Sesuvium portulacastrum (L.) 


\section{IMPORTANCE:}

The present study describes the phytochemical profile and antimicrobial activity of Sesuvium portulacastrum.

\section{MATERIALS AND METHODS:}

\section{Collection and validation of the samples:}

The leaves of Sesuvium portulacastrum were collected from the mangrove forests of Gilakaladindi, Mangrove estuary, Krishna District.

\section{Extract preparation:}

The amount of 100 grams of Sesuvium portulucastrum leaves powder was added to $125 \mathrm{ml}$ ethanol $96 \%$ or distilled water. The ethanolic and aqueous extracts mixture was preserved at laboratory temperature $25{ }^{\circ} \mathrm{C}$ for 48 hours and was stirred every few hours with a glass rod. The collecting supernatant was centrifuged by $9000 \mathrm{rpm}$ for $5-10 \mathrm{~min}$. The supernatant was removed and reached to the original volume with ethanol or distilled water, then the samples packed in dark containers and stored at refrigerator temperature after filtered by $0.45 \mu$ Whatman filter paper.

\section{Phytochemical analysis:}

Three extracts of $S$. portulacastrum obtained by extraction in aqueous, petroleum, diethylether solvents, respectively, were compared for their antimicrobial activity and extracts are further subjected to gas chromatography-mass spectrometry (GC-MS) analysis to find out the nature of the compounds responsible for the antimicrobial activity. The antibacterial activities were assessed by measuring the diameter of the inhibition zones, minimum inhibitory concentration (MIC) and minimum bactericidal concentration (MBC) values.

Phytochemical screening of the ethanolic extract of the leaves and stems of $S$. portulacastrum showed the presence of steroids.

While the aqueous extract was positive toward the presence of alkaloids, saponins, tannins and terpenoids. However, the dichloromethane extract was negative to all chemical tests done.

Presence of tested secondary metabolites in the leaves and stems of $S$. portulacastrum will be promising for further studies on the plant as a potential study area for other researchers.
The phytoconstituents detected in the plant materials could be responsible for the antimicrobial activity though their exact mode of action which is poorly understood up till now.

\section{RESULT:}

Compared to the aqueous and dichloromethane extract, the ethanolic extract showed better antimicrobial activity against Staphylococcus aureus and $E$. coli, indicating its potential application related to noscomial infections. GC-MS results revealed 22, 23-Dihydrostigmasterol, Benzoic acid, 3,4,5trihydroxy-(Gallic acid), (2R,3R)-(-)-Epicatechin and Capsaicin in the ethanolic extract to be the molecules responsible for the antimicrobial activity of $S$. portulacastrum.

\section{DISCUSSION \& CONCLUSION:}

The range of medicinal plants and herbs containing various phytochemicals with biological activity can be of therapeutic importance. Much of the protective effect of fruits and vegetables also has been attributed to the presence of phytochemicals. Different phytochemicals have been found to have a broad range of actions, which may help in protection and treatment against different diseases. In the present study, the leaves and stems of $S$. portulacastrum were subjected to phytochemical evaluation, where different solvent extracts used showed the detection of various phytochemical compounds through different chemical tests used based on their solubility. Phytochemical analysis with the use of GC-MS of the $S$. portulacastrum ethanolic extract revealed the presence of 22, 23-Dihydrostigmasterol, Benzoic acid 3, 4, 5-trihydroxy-, Epicatechin and Capsaicin. GCMS is used for preliminary identification of main chemical components of the plant extract. All of these compounds have been shown to have antibacterial activity.

\section{REFERENCES:}

1. Al-Azzawi A, Alguboori A, Hachim MY, Najat M, Al Shaimaa A and M Sad, 2012. Preliminary phytochemical and antibacterial screening of Sesuviam portulacastrum in the United Arab Emirates. Pharmacognosy Res., 4: 219-224

2. Chandrasekaran M, Senthilkumar A and V Venkatesalu. 2011. Antibacterial and antifungal efficacy of fatty acid methyl esters from the leaves of Sesuvium portulacastrum L. Eur. Rev. Med. Pharmacol. Sci. 7: 775780. 
3. Rabhi M, Giuntine D, Castagna A, Remorini D, Baldan B and A Smaoui, 2010. Sesuvium portulacastrum maintains adequate gas exchange, pigment composition and thylakoid proteins under moderate and high salinity, 2010. J. Plant Physiol. $16: 1336-41$.

4. Abirami, H. and R. Rameshwari: Antibacterial and antifungal screening of Sesuvium portulacastrum extracts against leather contaminating organism. Int. J .Curr. Res., 5, 1376 - 1377 (2013).

5. Chandrasekaran, M., A. Senthilkumar and V. Venkatesalu: Antibacterial and antifungal efficacy of fatty acid methyl esters from the leaves of Sesuvium portulacastrum. Eur. Rev. Med. Pharmacol. Sci., 15, 775-780 (2011).

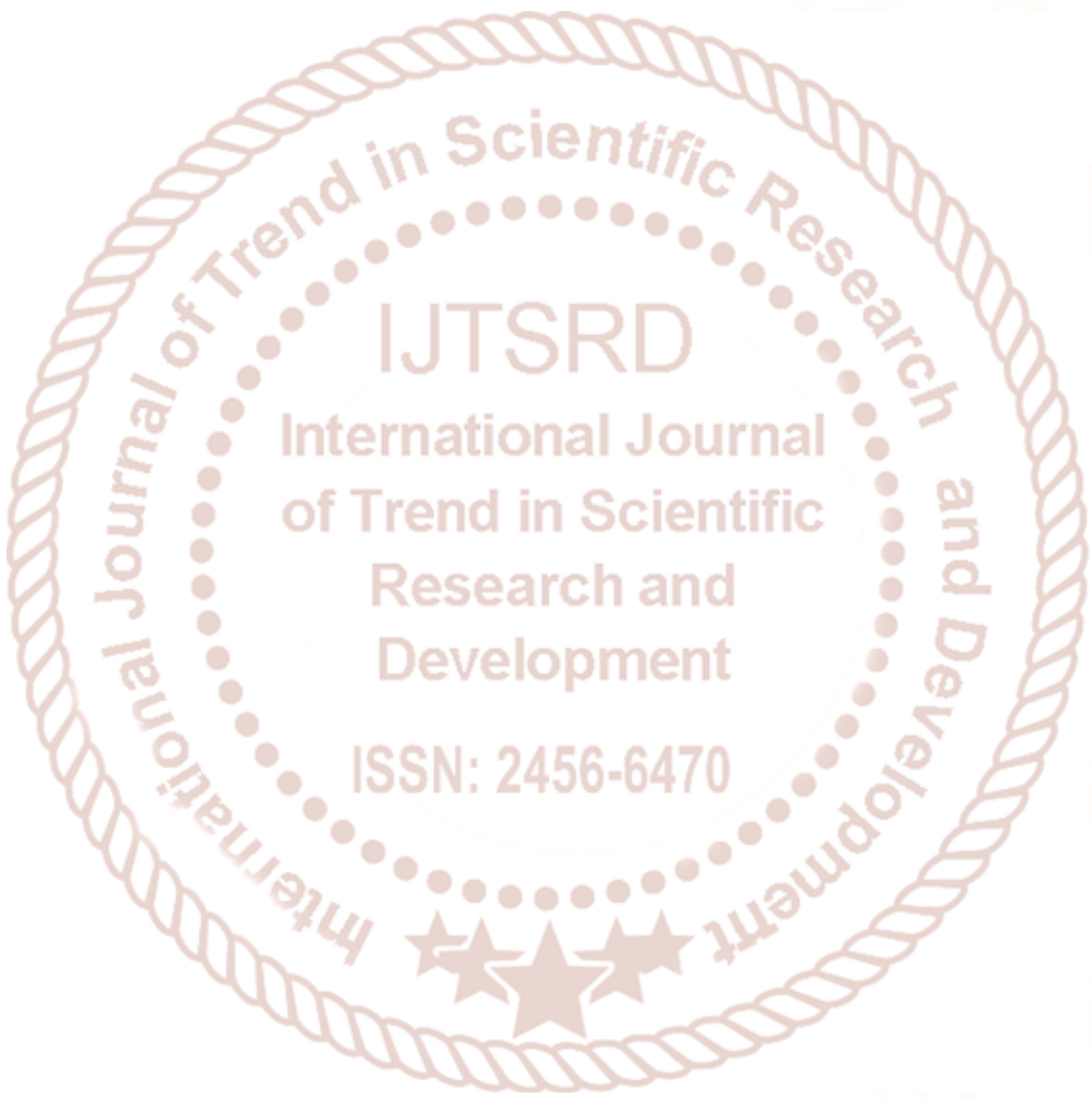

